\title{
The Scintillating Bubble Chamber (SBC) Experiment for Dark Matter and Reactor CEvNS
}

\author{
P. Giampa, for the SBC collaboration ${ }^{a, b, *}$ \\ ${ }^{a}$ TRIUMF, Vancouver, British Columbia, V6T 2A3, Canada \\ ${ }^{b}$ SNOLAB, Lively, Ontario, P3Y 1N2, Canada \\ E-mail: pgiampa@snolab.ca
}

\begin{abstract}
The Scintillating Bubble Chamber (SBC) experiment is a novel low-background technique aimed at detecting low-mass (0.7-7 GeV/c2) WIMP interactions and coherent scattering of reactor neutrinos (CEvNS). The detector consists of a quartz-jar filled with liquid Argon (LAr), which is spiked with $\mathrm{O}(100 \mathrm{ppm})$ of liquid Xenon (LXe) acting as a wavelength shifter. The target fluid is depressurized into a super-heated state by a mechanically controlled piston. Particles interacting with the LAr+100 ppm LXe can generate heat (bubbles) and scintillation light, depending on the energy intensity and density. The detector is further equipped with cameras to take pictures of the bubbles, Silicon-Photo-Multipliers to measure the scintillation light, and piezo-acoustic sensors to listen to the bubble's formation. By combining these observables, the SBC detector is aiming to reach a threshold for nuclear recoils of $100 \mathrm{eV}$ and a projected WIMP-sensitivity of 3.0x10-43 $\mathrm{cm}^{2}$, for a WIMP mass of $0.7 \mathrm{GeV} / \mathrm{c}^{2}$. In this paper, we will present the overall design of the SBC experiment and provide an update on the ongoing construction at the Fermilab site. Finally, we will discuss the collaboration's plans for the SNOLAB installation/operation and the reactor CEvNS search.
\end{abstract}

40th International Conference on High Energy physics - ICHEP2020

July 28 - August 6, 2020

Prague, Czech Republic (virtual meeting)

\footnotetext{
${ }^{*}$ Speaker
} 


\section{Introduction}

Bubble chambers, invented by D. A. Gasler in the early fifties [1], have played a significant role in the early days of particle physics because of their particle tracking abilities. Since then, particle tracking has significantly evolved, with new techniques such as time-projection-chambers and solid-state detectors. However, over the last decade, bubble chambers have been re-purposed as low-energy nuclear recoil detectors studying the nature of dark matter and neutrinos. [2] [3] [4]. A bubble chamber consists of a target fluid contained within a pressure-controlled volume. By slowly lowering the internal pressure, the target fluid is placed in a super-heated state, such that the saddle point in the Gibbs potential for the vapour state is lower than the liquid one. Consequently, if a particle interacting with the super-heated fluid generates sufficient localized heat to overcome the Seitz threshold [5], which is pressure dependent, it could lead to the nucleation of a vapour bubble. Therefore, the set fluid pressure, and temperature, determine the energy threshold of a bubble chamber. Moreover, the bubble formation process is also dependent on the incoming particle, with significant differences between electronics recoils (ER) and nuclear recoils (NR). Particles with low $d E / d x$ in the medium, such as $\gamma \mathrm{s}$ and $\beta$ s (ER), are less likely to generate enough localized heat compared to heavy-charged particles such as neutrons and $\alpha \mathrm{s}(\mathrm{NR})$, at equal threshold settings. Finally, after being imaged by an optical readout, the generated bubbles are removed by re-pressurizing the fluid volume, thus bringing the detector back to its starting state.

The SBC collaboration is currently developing a novel low-background bubble chamber that utilizes liquid argon (LAr) as a target fluid. The use of LAr enables the detector to operate at energy thresholds of the order of $100 \mathrm{eV}$ while retaining ER-blindness [17]. Moreover, switching to a liquid noble gas adds calorimetry capabilities via the scintillation process.

Given the properties of the SBC detector, the collaboration is currently pursuing two physics goals. Firstly, a low-mass $\left(<10 \mathrm{GeV} / \mathrm{c}^{2}\right)$ dark matter search focusing on Weakly Interacting Massive Particles (WIMPs), and secondly, a precision study of the reactor coherent elastic neutrino-nucleus scattering (CEvNS) interactions in argon and xenon. To achieve said goals, the collaboration is building two nearly identical detectors through a three-step program:

1. SBC-Fermilab: Build and commission the first SBC detector with a focus on the characterization, as a function of energy threshold, of the ER nucleation probability and the NR response.

2. SBC-SNOLAB: Build and install a second detector at the SNOLAB facility, Sudbury, ON, Canada, to complete a competitive low-mass WIMP search $\left(0.7-7 \mathrm{GeV} / \mathrm{c}^{2}\right)$. The experiment has a projected WIMP-sensitivity of $3.0 \times 10^{-43} \mathrm{~cm}^{2}$, for a WIMP mass of $0.7 \mathrm{GeV} / \mathrm{c}^{2}$ (Fig. 1).

3. SBC-CEvNS: Re-purpose and install the calibrated SBC-Fermilab detector at a reactor site for precision studies of CEvNS in LAr. The collaboration is currently investigating the Laguna Verde reactor site in Mexico as a possible location, with estimated rates displayed in Fig. 1. 

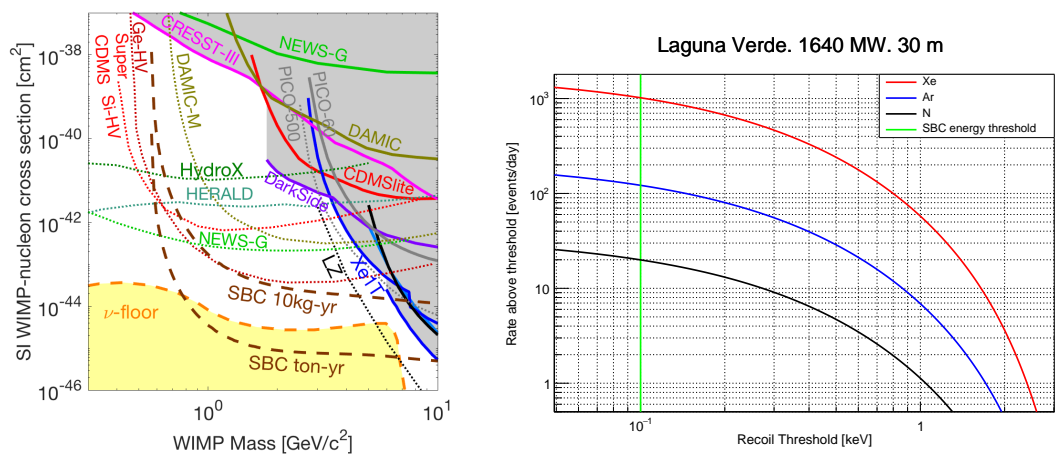

Figure 1: Left: Projected 90\% CL limits (in the absence of a dark matter signal) from $10 \mathrm{~kg}-\mathrm{yr}$ and 1 ton-yr exposures with future SBC detectors. Limits assume sensitivity to $100 \mathrm{eV}$ argon recoils. The 10 kg-yr limit assumes an observed background of one event, without subtraction. The ton-yr limit assumes the CEvNS background only, with background subtraction. The neutrino (CEvNS) floor is taken from [6]. Projections from SuperCDMS [7], LZ [8], DAMIC [15], HERALD (superfluid helium) [9], HydroX $\left(\mathrm{H}_{2}-\right.$ doped xenon) [10], NEWS-G and PICO (private communications) are also shown. The gray region indicates parameter space excluded by existing direct detection limits [4, 7, 11-16]. Right: Estimated CEvNS rate in xenon, argon and nitrogen, as a function of energy threshold, for the Laguna Verde reactor site in Mexico. For these simulations, the reactor power was set at $1640 \mathrm{MW}$, with the detector placed $30 \mathrm{~m}$ away.

\section{The SBC Experiment}

The current generation of SBC consists of $10 \mathrm{~kg}$ of LAr contained within two fused silica vessels, an inner and an outer jar. The LAr is doped with $\mathrm{O}(100 \mathrm{ppm})$ of Xe atoms to wavelength shift the VUV scintillation light from $128 \mathrm{~nm}$ to $175 \mathrm{~nm}$. This slight shift in emission peak enables the scintillation light to be transmitted through the fused silica. The jars are connected through bellows connection and controlled via a hydraulic system. The inner and outer jars, which are placed inside a stainless-steel pressure vessel $(\mathrm{PV})$, are surrounded by liquid $\mathrm{CF}_{4}\left(\mathrm{LCF}_{4}\right)$ which acts both as a thermal bath and hydraulic fluid. A piston is used to increase/decrease the pressure of the $\mathrm{LCF}_{4}$, moving the inner jar in/out of the outer jar. The pressure inside the PV cycles between 20 and 360 PSIA. To maintain a temperature difference between the target volume and the hydraulic area an HDPE insulation castle is installed inside the PV, which is cooled via closed-loop duel phase nitrogen thermosyphons. The $\mathrm{LCF}_{4}$ around the target area is cooled to $130 \mathrm{~K}$, while the $\mathrm{LCF}_{4}$ below the insulation castle is kept at a temperature of $90 \mathrm{~K}$. This thermal gradient is designed to ensure that nucleation can only occur in the instrumented portion of the LAr volume, with the conditions described above. $\mathrm{LCF}_{4}$ was identified as the hydraulic fluid as it is a stable liquid above $88 \mathrm{~K}$, between 20 and 360 PSIA. The PV is enclosed inside a vacuum jacket. A schematic and a rendering of the SBC detector are shown in Fig. 2.

The detector is equipped with three readout systems to image any nucleation, count any scintillation light and listen to the acoustics inside the jars. The bubble imaging is done by three 1 MP, $100 \mathrm{fps}$, OV9281 Omni Vision cameras controlled via microcontrollers, with a resolution of 1 MP. The three cameras are connected to the view-ports at the top of the PV via an optical system. Three Red LED rings, placed under the PV view-ports (Fig. 3), illuminate the chamber during bubble imaging. Moreover, the outer jar is wrapped in a Teflon reflector to increase light 


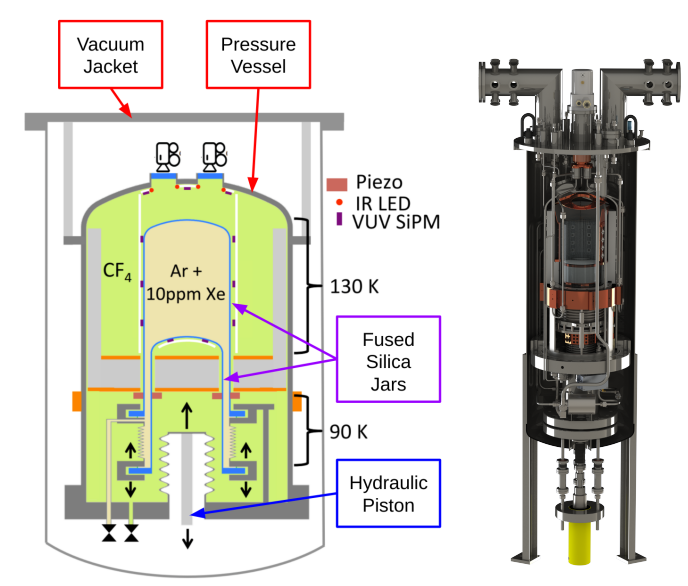

Figure 2: Left: schematic representation of the SBC detector with highlighted the fused silica jars, the hydraulic piston, the pressure vessel, the vacuum jacket and the readout systems. Right: 3D rendering of the fully assembled SBC detector.

collection. Scintillation light generated in the LAr is collected by 32 Hamamatsu VUV4 Quad Silicon-Photomultipliers (SiPMs) equally spaced onto the surface of the outer jar. The SiPMs are held in place by a 3D-printed holding structure that is spring-mounted onto the reflector surrounding the outer jar, as shown in Fig. 3. Finally, eight piezo acoustic sensors monitor the acoustics inside the jars. A spring-loaded system embedded in the HDPE insulation keeps the piezo sensors in contact with the outer jar, as illustrated in Fig. 3.

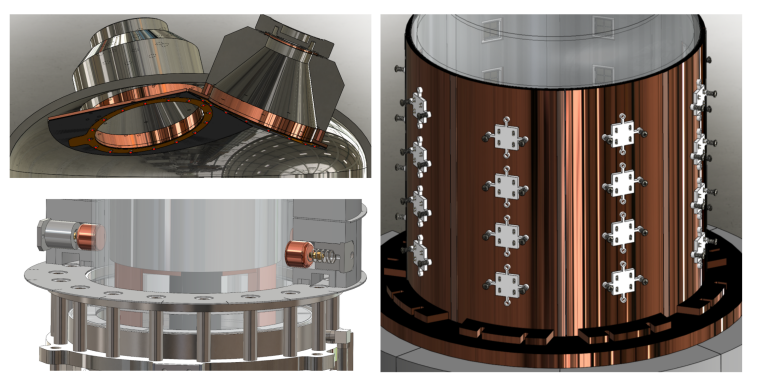

Figure 3: Top Left: Rendering of the PV view-ports and LED rings for the camera system. Bottom Left: Illustration of the piezo acoustic sensors mounted against the outer jar. Right: Drawing of the scintillation system, with the SiPMs mounted onto the reflector and viewing the LAr.

\section{Current Status and Outlook}

Constructions of the SBC-Fermilab chamber are already underway, with different subsystems already tested and installed. While the COVID-19 pandemic increases the uncertainty in the schedule, the collaboration expects the SBC-Fermilab detector to start its commission in the later part of 2021. The primary objectives for the Fermilab chambers are as follows: 
- Demonstrate the scalability of the techniques, following a first 30g Xenon scintillating bubble chamber prototype [17].

- Determine the probability of ER-induced nucleation as a function of threshold.

- Measure the nuclear recoil sensitivity of the chamber, using a ${ }^{9} \mathrm{Be}(\gamma, n)$ source [18] and other sources.

In the meantime, the collaboration is also moving forward with the second SBC chamber, optimized for a low-mass WIMP search. On-site activities for SBC-SNOLAB are currently ramping up through both infrastructure preparations and materials procurement. Moreover, the collaboration is currently undertaking a significant material screening program to select inner detector components with radioactivity levels consistent with the target background budget of one event per $10 \mathrm{~kg}$ per year. Installation of the SBC-SNOLAB chamber underground is expected to start in the second half of 2021, with the first physics data-taking campaign anticipated for the second portion of 2022. Lastly, after the aforementioned goals for SBC-Fermilab are achieved, the collaboration plans to move that particular detector to a reactor site where it can be re-purposed to study CEvNS in LAr and LXe. The SBC collaboration anticipates this reactor-site deployment following the conclusion of the SBC-Fermilab program.

\section{Conclusions}

The SBC collaboration is developing a novel bubble chamber technique that combines the ER-blindness capability of standard bubble chambers with the calorimetry abilities of liquid noble based detectors. With a target background budget of 1 event per $10 \mathrm{~kg}$ per year, this program will study both the nature of dark matter and coherent elastic neutrino-nucleus scatters in Ar and Xe. With these goals in mind, the SBC collaboration is developing two similar chambers via a three-step program : (1) SBC-Fermilab, dedicated to studying ER-induced nucleation and the NR response as a function of the threshold, (2) SBC-SNOLAB, a second chamber optimized for a low-mass WIMP search with projected sensitivity of $3.0 \times 10^{-43} \mathrm{~cm}^{2}\left(\mathrm{~m}_{\chi}=0.7 \mathrm{GeV} / \mathrm{c}^{2}\right)$, (3) SBC-CEvNS, upgrade and install (1) at a reactor site for precision studies of CEvNS in Ar and Xe. Constructions of the SBC-Fermilab chamber have already started, and data-taking is expected to begin in the latter part of 2021. In parallel, the collaboration is moving forward with the low-background SBC-SNOLAB detector, with construction anticipated to start in the first half of 2021 and first data-taking in 2022. SBC-CEvNS instead is expected to be installed at a reactor site once the SBC-Fermilab program is completed (Lacuna Verde reactor site in Mexico under consideration). Finally, the SBC collaboration is also investigating a possible next-generation tonne-scale scintillating bubble chamber.

\section{References}

[1] D. A. Glaser. "Some Effects of Ionizing Radiation on the Formation of Bubbles in Liquids", Phys. Rev. 87, 665 (1952). 
[2] Barnabé-Heider et al., "Response of Superheated Droplet Detectors of the PICASSO Dark Matter Search Experiment", Nucl. Instrum. Meth. A555 (2007) 184-204.

[3] E. Behnke et al., "First dark matter search results from a 4-kg CF3I bubble chamber operated in a deep underground site", Phys. Rev. D 90, 079902 (2014).

[4] C. Amole et al., "Dark Matter Search Results from the Complete Exposure of the PICO-60 C3F8 Bubble Chamber", Phys. Rev. D 100, 022001 (2019).

[5] F. Seitz, "On the Theory of the Bubble Chamber", The Physics of Fluids 1, 2 (1958).

[6] F. Ruppin, et al.,"Complementarity of dark matter detectors in light of the neutrino background",Phys. Rev. D 90, 083510 (2014).

[7] R. Agnese, et al., "Projected Sensitivity of the SuperCDMS SNOLAB experiment", Phys. Rev. D 95, 082002 (2017).

[8] D.S. Akerib, et al., "Projected WIMP sensitivity of the LUX-ZEPLIN dark matter experiment", Phys. Rev. D 101, 052002 (2020).

[9] S.A. Hertel, et al., "Direct detection of sub-GeV dark matter using a superfluid ${ }^{4} \mathrm{He}$ target", Phys. Rev. D 100, 092007 (2019).

[10] A. Monte for the HydroX collaboration, "R\&D towards hydrogen-doped LXe TPCs", APS DPF Meeting 2019.

[11] E. Aprile, et al., "Dark Matter Search Results from a One Ton-Year Exposure of XENON1T", Phys. Rev. Lett. 121, 111302 (2018).

[12] D.S. Akerib, et al., "Results from a search for dark matter in the complete LUX exposure", Phys. Rev. Lett. 118, 021303 (2017).

[13] P. Agnes, et al., "Low-Mass Dark Matter Search with the DarkSide-50 Experiment", Phys. Rev. Lett. 121, 081307 (2018).

[14] Q. Arnaud, et al., "First results from the NEWS-G direct dark matter search experiment at the LSM", Astropart. Phys. 97, 54-62 (2018).

[15] A. Aguilar-Arevalo, "Results on low-mass weakly interacting massive particles from a 11 kg-day target exposure of DAMIC at SNOLAB", arXiv, astro-ph.CO, 2007.15622 (2020).

[16] A.H. Abdelhameed, et al., "First results from the CRESST-III low-mass dark matter program", Phys. Rev. D 100, 102002 (2019).

[17] D. Baxter et al., "First Demonstration of a Scintillating Xenon Bubble Chamber for Detecting Dark Matter and Coherent Elastic Neutrino-Nucleus Scattering", Phys. Rev. Lett. 118, 231301 (2017).

[18] A. E. Robinson, "A reanalysis of radioisotope measurements of the ${ }^{9} \mathrm{Be}(\gamma, n)^{8} \mathrm{Be}$ crosssection", Phys. Rev. C 94, 024613 (2016). 